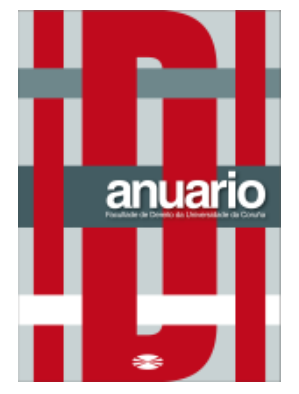

Anuario da Facultade de Dereito da Universidade da Coruña

Vol. 22 (2018), pp. 54-70

ISSNe: 2530-6324 || ISSN: 1138-039X

DOI: https://doi.org/afdudc.2018.22.0.5004

\title{
LA PROTECCIÓN DE LOS INMIGRANTES IRREGULARES EN LOS ESTADOS UNIDOS Y LA LIBERTAD DE CIRCULACIÓN EN UNA «UNIÓN AMERICANA»*
}

\author{
KRISTINA M. CAMPBELL \\ Catedrática de Derecho \\ Universidad del Distrito de Columbia \\ Washington, D.C. (Estados Unidos)
}

Resumen: Este artículo es una continuación y ampliación de una propuesta que formulé en 2009, y se plantea si - y cómo - los Estados Unidos pueden apartarse de su actual sistema de regulación de la inmigración punitivo, cuasi-penal y de exigencia dura de su cumplimiento. ¿Es posible para los Estados Unidos y sus vecinos acercarse a un sistema de protección de los refugiados y otros migrantes irregulares del resto de las Américas, modelado sobre el concepto de «libertad de circulación» de la Unión Europea? Si es así, ¿a qué debería parecerse dicho sistema? Permitiendo a todos los ciudadanos de una «Unión Americana» el derecho a viajar, vivir, y trabajar en América del Norte, sin los peligros inherentes al actual sistema de fronteras militarizadas de Estado-nación, ¿se forjarían nuevas y más fuertes alianzas entre los Estados Unidos, Canadá y México? ¿Puede - y debería - dicha «Unión Americana» expandirse más allá de los Estadosnación de América del Norte, para incluir el resto de las Américas, así como los ciudadanos América Central y América del Sur?

Palabras clave: emigración, inmigración irregular, libertad de circulación, protección, Unión Europea

\footnotetext{
*Agradezco al profesor Jesús Martínez Girón y al profesor Alberto Arufe Varela la invitación para hablar sobre este tema en el XII Congreso Internacional de Derecho Comparado del Trabajo, celebrado los días 25 y 26 de enero de 2018, en la Facultad de Derecho de la Universidad de A Coruña, España.
} 
Abstract: This article is a continuation and expansion of the proposal I put forth in 2009, and asks if - and how - the United States can move away from its current punitive, quasi-criminal, enforcement-heavy system of immigration regulation. Is it possible for the United States and its neighbors to move toward a system of protection for refugees and other irregular migrants from the rest of the Americas modeled on the European Union's concept of «Freedom of Movement»? If so, what would such a system look like? Would allowing all citizens of an «American Union» the right to travel, live, and work in North America without the dangers inherent in the current nation-state system of militarized borders forge new and stronger alliances between the United States, Canada, and Mexico? Can — and should - such an «American Union» be expanded beyond the nation-states of North America to include the rest of the Americas and the citizens of Central and South America?

Keywords: Migration, Irregular immigration, Freedom of movement, Protection, European Union

SUMARIO: I. INTRODUCCIÓN.- II. LA EVOLUCIÓN DEL DERECHO Y LA POLÍTICA DE INMIGRACIÓN EN LOS ESTADOS UNIDOS, DE 2009 HASTA LA ACTUALIDAD.- III. LA VIABILIDAD DE LA «LIBERTAD DE CIRCULACIÓN» EN UN CONTEXTO GLOBAL.- IV. LA CREACIÓN DE A UNA «UNIÓN AMERICANA».- 1. CANADÁ, MÉXICO Y LOS ESTADOS UNIDOS COMO UNA UNIÓN AMERICANA.- 2. LA INCLUSIÓN DE LAS NACIONES DE AMÉRICA CENTRAL Y SUDAMÉRICA EN UNA UNIÓN AMERICANA.- V. CONCLUSIÓN: EL FUTURO DEL ESTADO-NACIÓN.- BIBLIOGRAFÍA.

\section{INTRODUCCIÓN}

En estos tiempos turbulentos de migración global creciente, es claro que las vías por las cuales los Estados Unidos han estado tratando con la migración irregular no han producido cifras significativamente decrecientes de migrantes durante las pasadas dos décadas. En la historia reciente, no sólo el Gobierno federal ha hecho su política de inmigración más punitiva para todos los migrantes — regulares e irregulares-, sino que ha habido un movimiento de creciente implicación de los Estados federados en la regulación de la migración dentro de sus jurisdicciones ${ }^{1}$. En mi trabajo de 2009, «Imaginando una política de inmigración más humana en la era de Obama: el uso del

\footnotetext{
${ }^{1}$ Aunque la Corte Suprema de los Estados Unidos confirmó la supremacía del Gobierno federal para regular la inmigración en su decisión de Arizona v. United States en 2012, 567 U.S. _ (2012), los Gobiernos estatales y locales han continuado, sin embargo, intentando regular el Derecho inmigratorio dentro de sus jurisdicciones. Véase NATIONAL CONFERENCE OF STATE LEGISLATURES, «State Laws Related to Immigration and Immigrants», Junio 2017, disponible en http://www.ncsl.org/research/immigration/report-on-2017-state-immigration-laws-january-june.aspx («La legislación promulgada sobre inmigración se incrementó en la primera mitad de 2017 en un 90 por ciento, hasta 133 leyes, comparadas con las 70 leyes de 2016. El número de resoluciones se incrementó en un 22 por ciento, hasta 195, desde 159. Los legisladores de 47 Estados federados promulgaron 133 leyes y 195 resoluciones sobre inmigración, de un total de 328. Nueve proyectos más fueron vetados por los Gobernadores, y 18 están pendientes de firma» (énfasis en el original).
} 
poder plenario para detener la balcanización estatal de la regulación de la inmigración» ${ }^{2}$, argumentaba que dotar a los Estados federados de un poder incrementado para regular la inmigración, conduciría a un cuerpo de Derecho caótico e incongruente, y hacía la siguiente propuesta:

En mi opinión una interpretación radical de la llamada a incorporar los derechos humanos internacionales al Derecho y a la política de inmigración norteamericana puede conciliarse tanto con el deseo de los Estados Unidos de controlar nuestra soberanía, como con nuestra necesidad de una reforma completa de la inmigración. De hecho, creo que si hay que hacer una reforma migratoria significativa a nivel federal, los ramos políticos deben hacer más que modificar únicamente la INA — deben reescribirla, para reflejar lo que está convirtiéndose rápidamente en internacionalización del Derecho y la política de inmigración dentro de nuestras fronteras. Así, propongo que - tomando en préstamo la doctrina de la Corte Europea de Justicia sobre «libertad de circulación»- el Congreso y el Ejecutivo usen su poder plenario para regular la inmigración, reconstruyendo nuestras leyes y políticas de inmigración, de modo que se reconozca el derecho humano inherente de migrar, se ponga fin definitivamente a la descentralización del federalismo de la inmigración, y los Estados Unidos den un paso hacia adelante en la sociedad global en que vivimos en el siglo $\mathrm{XXI}^{3}$.

En retrospectiva, mi propuesta de que el Congreso y el Ejecutivo de los Estados Unidos «reconozcan el derecho humano inherente de migración», para adoptar nuestra «sociedad global» ${ }^{4}$, parece no sólo abiertamente optimista, sino ingenua. Sin embargo, a pesar de la multitud de vías por las cuales la migración global ha cambiado, continúo creyendo que es bueno para los Estados Unidos - y, verdaderamente, para el mundoseguir el ejemplo de la Unión Europea, y trabajar no hacia una política que debilite el principio de Estado-nación y sus fronteras, en vez de reforzar y fortificar dichas divisiones artificiales.

En cuanto tal, este artículo es una continuación y expansión de la propuesta que planteé en 2009, y se cuestiona si - y cómo - los Estados Unidos pueden salir de su sistema actual de regulación de la inmigración, punitivo, cuasi-criminal y de exigencia dura del cumplimiento. ¿Es posible para los Estados Unidos y sus vecinos avanzar hacia un sistema de protección de los refugiados y otros migrantes irregulares del resto de las Américas, modelado sobre el concepto de «libertad de circulación» de la Unión Europea? ${ }^{5}$ Si fuese así, ¿a qué debería parecerse dicho sistema? ¿Permitir a todos los

\footnotetext{
${ }^{2}$ Véase CAMBPELL, K.M., «Imagining a more humane immigration policy in the age of Obama: The use of plenary power to halt the State balkanization of immigration regulation», Saint Louis University Public Law Review, núm. 29 (2010), págs. 415 y ss.

${ }^{3}$ Ibidem, en 455.

${ }^{4}$ Ibidem, en 455-56.

${ }^{5}$ En la Unión Europea, existen dos conceptos distintos dentro de la expresión «libertad de circulación» libertad de circulación de personas y libertad de circulación de trabajadores. Véase GILBERT, J., Nomadic Peoples and Human Rights, Routledge (Londres, 2014), pág. 73; véase, también, GROENENDIJK, K., GUILD, E. y CARRERA, S. (Editores), Illiberal Liberal States: Immigration, Citizenship and Integration in the EU, Routledge (Londres, 2013), pág. 206. Este escrito considerará el derecho humano de libertad de circulación con carácter general, comprendiendo, en consecuencia, ambos conceptos dentro de la estructura de una potencial «Unión Americana».
} 
ciudadanos de una «Unión Americana» el derecho a viajar, vivir y trabajar en Norteamérica sin los peligros inherentes al actual sistema estatal-nacional de fronteras militarizadas forjaría nuevas y más fuertes alianzas entre los Estados Unidos, Canadá y México? ¿Puede - y debería- dicha «Unión Americana» expandirse más allá de los Estados-nación del Norte de América para incluir el resto de las Américas y los ciudadanos de América Central y del Sur?

Tras realizar un breve repaso de la evolución del Derecho de inmigración en los Estados Unidos entre el 2009 y el presente, abordaré por su turno cada una de esas cuestiones y propondré una hoja de ruta para abordar la migración irregular en las Américas en el siglo venidero.

\section{LA EVOLUCIÓN DEL DERECHO Y LA POLÍTICA DE INMIGRACIÓN EN LOS ESTADOS UNIDOS, DE 2009 HASTA LA ACTUALIDAD}

Tras la inauguración del mandato del Presidente Barack Obama en 2009, había muchas razones para ser optimista acerca de que una reforma de la inmigración significativa y amplia estaba en el horizonte. Esto fue particularmente cierto durante los dos primeros años de la Administración Obama, cuando el partido político del Presidente también controlaba ambas Cámaras del Congreso ${ }^{6}$. Si la decisión del Presidente y de los líderes del Congreso de no tocar la legislación inmigratoria se tomó con un exceso de confianza en su capacidad de seguir controlando el Gobierno en un futuro previsible, o por un deseo, en vez de ello, de centrarse en la cobertura sanitaria gubernamentalmente impuesta ${ }^{7}$, no importa mucho en retrospectiva. Ello es debido a que el resultado de la incomprensible inacción por parte de los líderes del Partido Demócrata a efectos de cumplir una de las principales promesas electorales del Presidente ha provocado la paralización del diálogo serio sobre una reforma inmigratoria amplia, dejando a los Estados Unidos y a sus líderes sin ideas para abordar lo que razonablemente se ha convertido en la cuestión definitoria de la presidencia de Obama, pero también de la de su sucesor, Donald J. Trump ${ }^{8}$.

\footnotetext{
${ }^{6}$ El Partido Demócrata tomó inicialmente el control del Congreso en las elecciones de 2006, antes de que Barack Obama se convirtiese en Presidente en 2008, pero perdió la mayoría en la Cámara de Representantes en 2010, y en el Senado en 2014. Véase, p. ej., ESTERBROOK, J., «Voters Usher Out Republicans», CBS News, 18 diciembre 2016, disponible en https://www.cbsnews.com/news/votersusher-out-republicans/; véase, también, CNN WIRE STAFF, «GOP Roars Back to Take U.S. House; Democrats Cling to Senate Majority», CNN, 3 noviembre 2010, disponible en http://www.cnn.com/2010/POLITICS/11/02/election.main/index.html; COLLINSON, S., «Republicans seize Senate, gaining full control of Congress», CNN, 5 noviembre 2014, disponible en http://www.cnn.com/2014/11/04/politics/election-day-story/index.html.

${ }^{7}$ Véase, p. ej., MURRAY, S. y MONTGOMERY, L., «House passes health-care reform bill without Republican votes», The Washington Post, 22 marzo 2010, disponible en http://www.washingtonpost.com/wp-dyn/content/article/2010/03/21/AR2010032100943.html.

${ }^{8}$ Desde que asumió el cargo en enero 2017, no ha habido supuestamente cuestión más utilizada por el Presidente Trump que la inmigración, tanto a efectos de unir a sus seguidores como de dividir a sus oponentes. La principal y más controvertida consecuencia de esto es, por supuesto, la de los repetidos esfuerzos de su Administración por promulgar una «prohibición de musulmanes» en la inmigración. Véase GONZALES, R., «Trump Travel Ban Allowed To Take Partial Effect», The Two Way: Breaking News from NPR, 13 noviembre 2017, disponible en https://www.npr.org/sections/thetwoway/2017/11/13/563923136/trump-travel-ban-allowed-to-take-partial-effect.
} 
No puede discutirse que Obama y la mayoría demócrata en el Congreso calcularon mal los riesgos de renunciar a aprobar una reforma inmigratoria antes de que perdieran su mayoría en la Cámara de Representantes en $2010^{9}$. Tras la estrecha derrota de la Ley del Sueño [americano] ${ }^{10}$ en 2010 en el Congreso - cuando cinco Senadores demócratas se juntaron a todos los Senadores republicanos para votar contra ella ${ }^{11}$-, el Partido Republicano no sólo hizo imposible reanimar una legislación progresiva como la de la Ley del Sueño [americano], sino que se embarcó en una agenda de reforzamiento de la exigencia del cumplimiento y expulsión de los no ciudadanos, a través de una miríada de propuestas legislativas a nivel federal ${ }^{12}$. Uniéndose al Congreso en su intento de promulgar leyes criminalizando y sancionando a los no ciudadanos - y en particular, a los inmigrantes irregulares en los Estados Unidos-, estuvieron los legisladores de diversos Estados federados, los cuales promulgaron durante la presidencia de Obama un sorprendente número de leyes estatales que buscaban regular la inmigración dentro de sus fronteras ${ }^{13}$. La más notoria de estas leyes estatales intentando regular la inmigración, S.B. 1070 de Arizona $^{14}$, acabó llegando a la Corte Suprema de los Estados Unidos en $2012^{15}$, y el grueso de la ley fue invalidado por la preeminencia del Derecho federal ${ }^{16}$.

Con Obama enfrentándose a la reelección en 2012, y un amplio número de Estados federados que continuaban afirmando su supuesto poder para regular por sí mismos la inmigración irregular, incluso tras la decisión de la Corte Suprema en el caso Arizona, el escenario para la batalla jurídica y política había quedado fijado. El 15 junio 2012 una fecha cuidadosamente elegida para honrar su significado histórico y simbólico, sobre la cuestión de la inmigración irregular ${ }^{17}$ - Obama anunció que haría uso de su

\footnotetext{
${ }^{9}$ Véase, p. ej., WEINER, R., «How immigration reform failed, over and over», The Washington Post, 30 enero 2013, disponible en https://www.washingtonpost.com/news/the-fix/wp/2013/01/30/howimmigration-reform-failed-over-and-over/?utm_term=.e16bbbb89dd4 («Como Bush, el Presidente Obama estaba cogido entre los votantes hispanos y el GOP. En 2009, él declaró la reforma inmigratoria una prioridad, pero reconoció que había demasiado en este plato como para hacerlo rápido»). También, véase YELLIN, J., «Obama not pushing to pass immigration reform this year», CNN, 10 abril 2009, disponible en http://www.cnn.com/2009/POLITICS/04/09/obama.immigration/.

10 S. 3992, Ley DREAM (Desarrollo, Socorro y Education de Menores Extranjeros) de 2010, $111^{\text {th }}$ Congress (2009-2010), disponible en https://www.congress.gov/bill/111th-congress/senate-bill/3992.

11 Véase WONG, S. y TOEPLITZ, S., «DREAM Act Dies in Senate», Politico, 20 diciembre 2010, disponible en https://www.politico.com/story/2010/12/dream-act-dies-in-senate-046573 («[C]inco demócratas votaron contra la legislación: Kay Hagan de Carolina del Norte, Mark Pryor de Arkansas, Ben Nelson de Nebraska y los dos demócratas de Montana, Jon Tester y Max Baucus»).

12 Véase, p. ej., «Why immigration reform died in Congress», NBC News, disponible en https://www.nbcnews.com/politics/first-read/why-immigration-reform-died-congress-n145276 («[A]hora, ninguna persona razonable puede decir que la muerte de la inmigración —en 2013 y 2014 - es culpa de nadie salvo de los Republicanos en la Cámara»).

13 A comienzos de 2012, un estudio afirmó que 164 leyes estatales y locales sobre inmigración se aprobaron, solamente en 2010 y 2011. Véase GORDON, I. y RAJA, T., «164 Immigration Laws Passed Since 2010? A MoJo Analysis», Mother Jones, marzo/abril 2012, disponible en http://www.motherjones.com/politics/2012/03/anti-immigration-law-database/\#.

${ }_{14}$ AZ Senate Bill 1070 (2010), disponible en https://www.azleg.gov/legtext/49leg/2r/bills/sb1070s.pdf.

${ }^{15}$ Arizona v. United States, 567 U.S. __ (2012).

${ }^{16}$ Ibidem.

${ }^{17}$ DACA se anunció en el $30^{\circ}$ aniversario de la decisión por la Corte Suprema de los Estados Unidos de Plyler v. Doe, 457 U.S. 202 (1982), que estableció el derecho de los niños indocumentados a asistir a las escuelas públicas en los grados K-12, sin pagar matrícula o tasas, con independencia de su estatus inmigratorio. Véase PRESTON, J. y CUSHMAN, Jr., J.H., «Obama to Permit Young Migrants to Remain in U.S.», The New York Times, 15 junio 2012, disponible en http://www.nytimes.com/2012/06/16/us/us-
} 
poder ejecutivo para ordenar a su Ministra de Seguridad Interior, Janet Napolitano, que siguiese una nueva política de exigencia del cumplimiento denominada «Acción Diferida para las Llegadas de Niños» $(\mathrm{DACA})^{18}$. El programa DACA prevé que los inmigrantes irregulares que llegaron como niños a los Estados Unidos ${ }^{19}$, que hubiesen completado o prosiguiesen una formación continua y que no tuviesen ninguna condena penal «significativa» ${ }^{20}$, pudiesen solicitar y recibir un permiso temporal para trabajar legalmente en los Estados Unidos ${ }^{21}$. Además, asumiendo que no eran deportables desde los Estados Unidos por ninguna razón distinta de su estatus de inmigrantes irregulares, los participantes en el programa DACA tenían derecho a que se difiriese el comienzo de los procedimientos de deportación, o a que los procedimientos pendientes fuesen administrativamente cerrados por la Administración de Inmigración y Aduanas (ICE) ${ }^{22}$.

Tras el anuncio de la creación del programa DACA, Obama caminaba a ser reelegido en noviembre de 2012, debido en parte significativa al apoyo de la comunidad latina de los Estados Unidos ${ }^{23}$. El programa DACA fue ensalzado por los defensores de los derechos de los inmigrantes y por otros que buscan ayuda frente a la deportación y la seguridad del permiso de trabajo para algunos de los millones de inmigrantes irregulares que, a ojos de muchos, merecían protección, porque fueron traídos a los Estados Unidos cuando eran niños por sus padres o por otros tutores adultos ${ }^{24}$. El argumento de que los beneficiarios de DACA, también conocidos como «soñadores» ${ }^{25}$, eran víctimas inocentes del sistema de inmigración y eran, en consecuencia, más meritorios que otros inmigrantes irregulares, no ha estado a salvo de críticas ${ }^{26}$. Sin embargo, hubo un amplio apoyo para el principio de que a los adultos jóvenes que han empleado la mayor parte de sus vidas en los Estados Unidos, y a menudo no tienen recuerdos ni raíces en sus países

to-stop-deporting-some-illegal-immigrants.html («Funcionarios de la Casa Blanca dijeron que elegían el viernes para anunciar la medida, por causa de que era el $30^{\circ}$ aniversario de la decisión de la Corte Suprema, Plyler v. Doe, que efectivamente estableció que todos los niños, con independencia de su estatus inmigratorio, tenían derecho a la educación pública en la escuela secundaria»).

18 DACA fue llevada a cabo a través de un memorandum político promulgado por la Ministra del Ministerio de Seguridad Interior, Janet Napolitano. Véase Memorandum, «Exercising Prosecutorial Discretion with Respect to Individuals Who Came to the United States as Children», 15 junio 2012, disponible en https://www.dhs.gov/xlibrary/assets/s1-exercising-prosecutorial-discretion-individualswho-came-to-us-as-children.pdf.

${ }^{19}$ Ibidem.

${ }^{20}$ Ibidem.

${ }^{21}$ Ibidem.

${ }^{22}$ Ibidem.

${ }^{23}$ Véase, p. ej., RODRIGUEZ, C.Y., «Latino vote key to Obama’s re-election», CNN, 9 noviembre 2012, disponible en http://www.cnn.com/2012/11/09/politics/latino-vote-key-election/index.html.

${ }^{24}$ Véase, p. ej., SHERRY, A., «Illegal-immigrant children brought to U.S. get break from deportation», The Denver Post, 15 junio 2012, disponible en http://www.denverpost.com/2012/06/15/illegal-immigrantchildren-brought-to-u-s-get-break-from-deportation/.

25 Véase, p. ej., «What is Daca and who are the Dreamers», The Guardian, disponible en https://www.theguardian.com/us-news/2017/sep/04/donald-trump-what-is-daca-dreamers [ «El programa DACA fue un compromiso ideado por la Administración Obama después de que el Congreso se negase a aprobar la llamada Ley de Desarrollo, Socorro y Educación de Menores Extranjeros (Dream), que habría ofrecido a aquellos que llegaron ilegalmente siendo niños, la oportunidad de una residencia legal permanente»).

${ }^{26}$ Véase, p. ej., SATI, J., «How DACA pits "good immigrants" against millions of others», The Washington Post, 7 septiembre $2017, \quad$ disponible https://www.washingtonpost.com/news/posteverything/wp/2017/09/07/how-daca-pits-good-immigrantsagainst-millions-of-others/?utm term $=.85 \mathrm{~b} 6 \mathrm{f} 446689 \mathrm{~b}$. 
de origen, debería dárseles la oportunidad de regularizar su estatus de inmigrantes y de convertirse en miembros plenos en nuestra sociedad ${ }^{27}$.

Inicialmente, el programa DACA y el propio movimiento de los Soñadores fue bastante exitoso, con aproximadamente 800.000 personas con derecho al DACA solicitando sus beneficios $\mathrm{y}$ «saliendo de las sombras» ${ }^{28}$. El lema del movimiento, «Indocumentados y sin miedo» ${ }^{29}$, empujó a mucha gente con derecho a DACA conscientemente a girar hacia un gran acuerdo de información personal con el Gobierno, en orden a percibir los beneficios que se derivaban del estatus coloquial de ser «DACAmentados» ${ }^{30}$. Sin embargo, se estaba cociendo un contragolpe, y la estrategia para desmantelar DACA se inició en 2015, cuando 26 Estados federados plantearon demanda en Texas contra el Gobierno federal, alegando que el Presidente Obama se había excedido en su poder ejecutivo cuando creó la política DACA en 2012, y su intento subsiguiente de crear una política paralela para los padres de los ciudadanos de los Estados Unidos sin estatus de inmigración legal en $2014^{31}$. Tras haber sido suspendida la ley por el Juez Andrew Hanen en Texas y confirmado ello por la Corte de Apelaciones del Quinto Circuito de los Estados Unidos ${ }^{32}$, la impugnación constitucional del programa DACA alcanzó por fin la Corte Suprema de los Estados Unidos en 2016, en la que los jueces empataron 4 a 4 y la decisión del Quinto Circuito se mantuvo ${ }^{33}$.

Tras la elección de Donald Trump como Presidente de los Estados Unidos, el espíritu del programa DACA cayó en barrena, y el Fiscal General Jeff Sessions anunció en septiembre de 2017 que se pondría fin al programa DACA al inicio de $2018^{34}$. Entre tanto, el Partido Republicano mantiene el control de ambas Cámaras del Congreso de los Estados Unidos, y ha estado diseñando un gran acuerdo legislativo orientado a exigir el cumplimiento del Derecho inmigratorio federal contra los inmigrantes irregulares, así

\footnotetext{
27 Véase, p. ej., TREVINO, R., «DACA and "dreamers" worthy of support», USA Today, 30 octubre 2017, disponible en https://www.usatoday.com/story/opinion/contributors/2017/10/30/daca-anddreamers-worthy-support/807131001/.

${ }^{28}$ Véase KRISHNAKUMAR, P., FOX, J. y LEVINE, A., «What's next for DACA and the nearly 800,000 people protected by it», Los Angeles Times, 6 septiembre 2017, disponible en http://www.latimes.com/projects/la-na-pol-daca-future/.

${ }^{29}$ La frase ha sido utilizada como un eslogan y lema activista para organizar a los jóvenes indocumentados, tanto antes como después de la puesta en práctica de DACA en 2012. Véase, p. ej., «Are you undocumented and unafraid», Dream activist, disponible en http://action.dreamactivist.org/callout.

${ }^{30}$ Poco después de la aprobación de DACA, los beneficiarios de DACA y los activistas acuñaron el término «DACAmentados» para describir su estatus como inmigrantes. Véase, p. ej., AMERICAN IMMIGRATION COUNCIL, «How DACA is Impacting the Lives of Those Who are Now DACAmented», Informe especial, 15 agosto 2013, disponible en https://www.americanimmigrationcouncil.org/research/how-daca-impacting-lives-those-who-are-nowdacamented.

${ }^{31}$ Véase FOLEY, E., «Over Half the States are Suing Obama For Immigration Actions», The Huffington Post, 25 enero 2015, disponible en https://www.huffingtonpost.com/2015/01/26/states-lawsuitimmigration_n_6550840.html.

${ }^{32}$ Véase FORD, M., «A Ruling Against the Obama Administration on Immigration», The Atlantic, 10 noviembre 2015, disponible en https://www.theatlantic.com/politics/archive/2015/11/fifth-circuit-obamaimmigration/415077/.

${ }^{33}$ Véase WHEELER, L. y FABIAN, J., «Deadlocked Supreme Court blocks Obama on immigration», The Hill, 23 junio 2016, disponible en http://thehill.com/regulation/court-battles/president-obamaimmigration-actions-programs-blocked-supreme-court-deadlocked-scalia-dapa-daca-crushing-blow.

${ }^{34}$ Véase SHEAR, M.D. y DAVIS, J.H., «Trump Moves to End DACA and Calls on Congress to Act», The New York Times, 5 septiembre 2017, $\quad$ disponible en https://www.nytimes.com/2017/09/05/us/politics/trump-daca-dreamers-immigration.html.
} 
como a una legislación imponiendo la cooperación de las policías locales con las autoridades federales de inmigración, y castigando a aquellas jurisdicciones estatales y locales que tuviesen políticas de santuarios en los que poder colocar a los inmigrantes irregulares en sus comunidades ${ }^{35}$.

El triste legado de la Administración Obama es que la promesa de una reforma inmigratoria amplia realizada al comienzo de su presidencia, no solamente fracasó en su realización, sino que el estatus de los inmigrantes irregulares en los Estados Unidos es ahora incluso más vulnerable. La hostilidad a la creación de DACA por el Presidente Obama, y los continuos esfuerzos del Congreso para desnudar la ley de protecciones procedimentales para los niños extranjeros no acompañados (UACs) ${ }^{36}$, así como el creciente tratamiento hostil de los solicitantes de asilo en la frontera de los Estados Unidos con México ${ }^{37}$, ha conducido a muchos inmigrantes irregulares a buscar protección dirigiéndose en busca de refugio a otros países, tales como Canadá ${ }^{38}$. De este modo, la política y el Derecho inmigratorios de los Estados Unidos ha regresado hacia una creciente criminalización, en lugar de evolucionar hacia una mayor fluidez y construcción de la nación, desde que Obama asumió el cargo en 2009.

\section{LA VIABILIDAD DE LA «LIBERTAD DE CIRCULACIÓN» EN UN CONTEXTO GLOBAL}

La idea central del concepto de libertad de circulación en la Unión Europea empezó con la idea de que los ciudadanos de los Estados miembros de la UE deberían ser capaces de buscar trabajo allí donde estuviese disponible, con la finalidad de buscarse su sustento ${ }^{39}$. La creencia de que el derecho al trabajo y a sustentarse a sí mismo y a su propia familia es un derecho humano, es crucial para el desarrollo y la sostenibilidad de la Unión Europea como un ideal y un modelo para otras naciones que deseen crear uniones similares entre sus Estados-nación vecinos ${ }^{40}$.

En la Unión Europea, todas las personas de los Estados miembros tienen derecho a la «libertad de circulación», que inicialmente se refería a los derechos de las personas a

35 H.R. 3003, «No Sanctuary for Criminals Act», $115^{\text {th }}$ Congress (2017-2018), disponible en https://www.congress.gov/bill/115th-congress/house-bill/3003. También, véase CLARK, D., «House Passes "Kate's Law", Votes to Defund Sanctuary Cities», NBC News, 29 junio 2017, disponible en https://www.nbcnews.com/politics/immigration/house-immigration-crackdown-n778141.

${ }^{36}$ Véase MASON, J., «Trump seeks border wall, crackdown on unaccompanied minors for "Dreamer" deal», Reuters, 8 octubre 2017, disponible en https://www.reuters.com/article/us-usa-immigrationexclusive/trump-Véaseks-border-wall-crackdown-on-unaccompanied-minors-for-dreamer-dealidUSKBN1CD10L.

${ }^{37}$ Véase, p. ej., «US Border Patrol Violates Human Rights of 40\% of Mexicans», Telesur, 22 septiembre 2017, disponible en https://www.telesurtv.net/english/news/US-Border-Patrol-Violates-Human-Rights-of40-of-Mexicans-20150922-0004.html.

${ }^{38}$ Véase, p. ej., «Refugees continue to cross from US to Canada in unprecedented numbers», Fox News, 5 octubre 2017, disponible en http://www.foxnews.com/world/2017/10/05/refugees-continue-to-cross-fromus-to-canada-in-unprecedented-numbers.html.

${ }^{39}$ Véase EUROPEAN COMMISSION, «Freedom to live and move in Europe: A guide to your rights as an EU citizen», EU Law and Publications, 13 octubre 2017, disponible en https://publications.europa.eu/en/publication-detail/-/publication/51cf67c4-a4ed-44a6-93cf8120a7aa4c08/language-en/format-PDF/source-search.

${ }^{40}$ Ibidem. 
migrar dentro de Europa, con la finalidad de obtener trabajo y sustento para sus familias ${ }^{41}$. El concepto de «libertad de circulación» fue eventualmente interpretado para incluir los derechos de las personas a entrar en un país, y establecía limitaciones sobre cuándo y cómo la residencia de una persona podía finalizarse por el Estado-nación ${ }^{42}$. Este compromiso con la «libertad de circulación» también condujo a los Estados miembros de la UE a luchar por políticas comunes y complementarias de inmigración entre los Estados-nación, con un particular énfasis no sólo en cómo abordar la inmigración irregular, sino también la entrada, residencia, retorno y expulsión de los no ciudadanos $^{43}$.

El principio de la «libertad de circulación» y la descentralización de competencias sobre las fronteras en la UE, ha sido gravemente desafiado últimamente, por causa de las preocupaciones sobre el terrorismo y el incremento de refugiados de Siria y de otras nacIones sacudidas por la guerra de Oriente Medio y del Norte de África ${ }^{44}$. Tras el ataque terrorista del Estado Islámico de Irak en el Levante (ISIL) en París, Francia, en noviembre 2015, las fronteras entre los Estados-nación de la Unión Europea se cerraron por vez primera desde la aprobación del Tratado Schengen ${ }^{45}$. Además, el creciente flujo de refugiados que escapaban del caos civil y de la guerra en Siria desde $2011^{46}$, han conducido al cierre de fronteras internacionales dentro de los Estados miembros de la UE, y a la negativa por los Estados miembros a aceptar refugiados de Estados no miembros de la UE, como resultado de ello ${ }^{47}$.

Dejando a un lado el potencial incumplimiento del principio de non-refoulemont ${ }^{48}$ del Derecho Internacional a que tales acciones daban lugar, el desafío a la viabilidad de un

\footnotetext{
${ }^{41}$ Véase EUROPEAN COMMISSION, «Freedom to move and live in Europe: A Guide to your rights as an EU citizen», EU Law and Publications, 17 octubre 2013, disponible en https://publications.europa.eu/en/publication-detail/-/publication/51cf67c4-a4ed-44a6-93cf-

8120a7aa4c08/language-en/format-PDF/source-search, en $6[\ll E l$ artículo 21(1) del Tratado de Funcionamiento de la Unión Europea establece que todo ciudadano de la UE tiene derecho a circular y residir libremente dentro del territorio de los países de la UE, con sujeción a las limitaciones y condiciones señaladas en los Tratados y a las medidas adoptadas para darles efecto»].

${ }^{42}$ Ibidem.

${ }^{43}$ Ibidem.

${ }^{44}$ Véase, p. ej., INTERNATIONAL RESCUE COMMITTEE, «Refugee Crisis: Europe and Middle East», disponible en https://www.rescue.org/topic/refugee-crisis-europe-middle-east («Más gente ha sido obligada a escapar de sus hogares por el conflicto y la crisis que en ningún período desde la II Guerra Mundial»).

45 Véase «Schengen Area», European Commission, Migration and Home Affairs, disponible en https://ec.europa.eu/home-affairs/what-we-do/policies/borders-and-visas/schengen_en (última actualización, 12 enero 2017); también, véase HATTEM, J., «France declares state of emergency, closes borders after attacks», The Hill, 13 noviembre 2015, disponible en http://thehill.com/policy/nationalsecurity/260134-france-declares-state-of-emergency-closes-borders-after-attacks.

46 Véase «Syria: The Story of the Conflict», BBC News, 11 marzo 2016, disponible en http://www.bbc.com/news/world-middle-east-26116868.

47 Véase MOHDIN, A., «These are the routes being closed off to refugees fleeing into Europe», Quartz, 10 marzo 2016, disponible en https://qz.com/635110/these-are-the-routes-being-closed-off-to-refugeesfleeing-into-europe/ («Diversos países —incluidos algunos de los Estados balcánicos- están cerrando algunas de las sendas que los traficantes han explotado para traer gente a la UE. En el último mes, algunos países europeos han cerrado o restringido duramente las principales rutas usadas por cientos de miles de refugiados para entrar en la UE. La Canciller alemana Angela Merkel ha condenado a los países europeos por cerrar "unilateralmente" la rutas balcánicas a los refugiados»).

${ }^{48}$ Véase UNITED NATIONS HIGH COMMISSIONER ON REFUGEES, «Note on Non-Refoulement (Submitted by the High Commissioner)», disponible en http://www.unhcr.org/en-
} 
sistema modélico en marcha de «libertad de circulación» para el mundo, es claro. ¿Cuándo los derechos de la gente a migrar pasan a un segundo plano, frente a la seguridad y otras preocupaciones del Estado-nación? ¿Es el derecho humano a la migración algo que pueda comprometerse, o es una libertad fundamental sobre la que los Estados-nación deberían trabajar juntos para asegurar que se proteja? ¿O es todo el concepto de «libertad de circulación» una idea radical que es inalcanzable en nuestro mundo moderno?

A pesar de la incerteza que rodea a la viabilidad del concepto de relajar o erradicar las fronteras entre Estados-nación, en este artículo, propongo que no sólo es posible el reconocimiento del concepto de «libertad de circulación»en un contexto global, sino que es necesario que avancemos en el intento de resolver nuestros problemas con la inmigración irregular y la migración forzada. En mi opinión, el único camino a través del que los Estados-nación pueden retener su soberanía y respetar los derechos humanos fundamentales de todas las personas es el de adoptar el concepto de «libertad de circulación», y trabajar hacia leyes de asilo e inmigración complementarias en los diversos Estados-nación. En el hemisferio occidental, propongo que esto significa la creación de una «Unión Americana», que no sea igual que la Unión Europea, pero que se oriente a abordar los problemas específicos de la inmigración irregular y de la migración forzada en las Américas.

\section{LA CREACIÓN DE A UNA «UNIÓN AMERICANA»}

A comienzos del siglo XXI, la Unión Europea se ha enfrentado a una crisis como no se había visto desde su creación. Al ocurrir la guerra civil de Siria en 2011, Europa ha experimentado el mayor flujo de refugiados y de personas desplazadas desde la II Guerra Mundial ${ }^{49}$. Este flujo «sin precedentes» de refugiados y de otras personas desplazadas por la guerra y por el conflicto en Oriente Medio y en el norte de África, ha causado que se cuestione de algún modo tanto el deseo como el futuro del concepto conjunto de una «Unión Europea» ${ }^{50}$. Así, el reciente flujo de personas que buscan protección de los Gobiernos de los Estados miembros desde el resto del mundo, ha conducido a cuestionarse gravemente la erradicación de fronteras dentro de los Estados miembros y la capacidad de dichos Estados miembros para absorber el gran número de refugiados que buscan seguridad dentro de la $\mathrm{UE}^{51}$.

us/excom/scip/3ae68ccd10/note-non-refoulement-submitted-high-commissioner.html [«Ningún Estado contratante expulsará o devolverá ("refouler") un refugiado de ningún modo a las fronteras de aquellos territorios en donde su vida o libertad estarían amenazadas por causa de su raza, religión, nacionalidad, pertenencia a un grupo social particular u opinión política»).

${ }^{49}$ Véase WESTCOTT, L., «The World's 'Unprecedented' Refugee Crisis By the Numbers», Newsweek, 18 junio 2015, disponible en http://www.newsweek.com/worlds-unprecedented-refugee-crisis-numbers344171.

${ }^{50}$ Véase DEARDEN, L., «Refugee Crisis: Concern over "unprecedented" arrivals in Greece and Italy after 2016 total passes 100,000», Independent, 23 febrero 2016, disponible en http://www.independent.co.uk/news/world/europe/refugee-migrant-crisis-concern-unprecedentednumbers-greece-italy-2016-passes-100000-a6891101.html.

${ }^{51}$ Ibidem. 
Cuando escribo esto, aunque no han ocurrido cambios en la política oficial en relación con la amplia circulación sin restricciones de personas dentro de los Estados miembros de la UE, parecen inevitables cambios en la regulación y estructura actuales que gobiernan el movimiento de personas dentro de la UE. Esto es debido no sólo al gran número de refugiados que buscan un lugar seguro en la UE, sino también a las tácticas actuales de las organizaciones terroristas globales. Los grupos terroristas radicales, como el ISIL, se dirigen a ciudadanos enfadados y a residentes en los Estados miembros de la UE, para reclutarlos, afiliarlos, y ejecutar ataques contra la población civil de la $\mathrm{UE}^{52}$.

La potencial capacidad de los miembros de grupos terroristas para cruzar las fronteras de los Estados miembros de la UE sin ningún tipo de vigilancia, ha cuestionado de algún modo si la falta de policía de fronteras debería alterarse o erradicarse completamente en nombre de la seguridad global ${ }^{53}$. En cuanto tal, la noción del modelo de la Unión Europea de una coalición cooperativa de Estados-nación ha dejado de ser concebida más o menos como un objetivo realista para el resto del mundo ${ }^{54}$. Sin embargo, la posibilidad de crear uniones continentales adicionales con controles fronterizos relajados - y en particular, una «Unión Americana»—, como respuesta a la crisis global de refugiados, se mantiene como una idea digna de ser considerada, con independencia de los obstáculos que permanecen en el camino para hacer de dicho empeño una realidad.

\section{CANADÁ, MÉXICO Y LOS ESTADOS UNIDOS COMO UNA UNIÓN AMERICANA}

La historia de la migración transnacional entre los tres países que actualmente comprenden América del Norte está llena de complicaciones ${ }^{55}$. En años recientes, ha habido mucha tensión entre los Estados Unidos y México, en relación con la migración irregular en la frontera sur de los Estados Unidos, y el supuesto papel que México juega facilitando el tránsito continuo de inmigrantes irregulares a los Estados Unidos ${ }^{56}$. Más recientemente, con la elección de Donald Trump como Presidente de los Estados Unidos en 2016, se ha prestado mucha atención a su promesa de construir un muro en la

\footnotetext{
52 Véase ROGERS, J., «Europe flooded with over 1,700 ISIS terrorists primed to attack, EU reveals», Express, 9 diciembre 2016, disponible en https://www.express.co.uk/news/world/741587/ISIS-terroristsEU-Europe-flooded.

53 Véase O'SUlLIVAN, F., «The Fading Dream of a Borderless Europe», Citylab, 13 enero 2017, disponible en https://www.citylab.com/equity/2017/01/the-fading-dream-of-a-borderless-europe/512816/.

${ }^{54}$ Ibidem. «Una Unión Europea caracterizada por fronteras internas abiertas bien puede ser remodelada en un futuro cercano. Pero con unos beneficios del sistema todavía tan grandes - y el proceso para deshacerlo tan complejo y costoso-, no es probable que desaparezca por completo en breve tiempo».

${ }^{55}$ Desde el siglo XV, toda América del Norte ha sido colonizada y sujeta diversamente a las normas francesas, españolas, holandesas y británicas. Véase, p. ej., KUPPERMAN, K.O., North America and the beginnings of European colonization, American Historical Association (Washington, D.C., 1992).

${ }^{56}$ Véase MCKINLEY Jr., J.C., «A Mexican Manual for Illegal Migrants Upsets Some in U.S.», The New York Times, 6 enero 2005, disponible en http://www.nytimes.com/2005/01/06/world/americas/a-mexicanmanual-for-illegal-migrants-upsets-some-in-us.html.
} 
frontera México-Estados Unidos, que supuestamente serviría como barrera impenetrable de los Estados Unidos sin autorización legal ${ }^{57}$.

Por causa del carácter díscolo entre los Estados-nación de América del Norte - y en particular, México y los Estados Unidos-, parece improbable que una unión de América del Norte con controles fronterizos relajados sea una posibilidad seria. Sin embargo, afirmo que una alianza al estilo de la UE entre Canadá, México y los Estados Unidos podría servir para reducir la migración irregular y facilitar controles fronterizos eficientes entre los Estados-nación ${ }^{58}$. Esto posibilitaría que los Estados-nación de América del Norte se centrasen más en la exclusión de personas que pueden presentar una amenaza terrorista en sus fronteras u otra intención maliciosa, aunque permitiendo también que las personas que simplemente deseen trabajar o disfrutar de otro modo de los beneficios de la residencia en un Estado-nación contiguo de América del Norte, tengan la capacidad de hacerlo sin miedo a la deportación inminente.

Sin embargo, a pesar del hecho de que pueda ser ventajoso en muchos aspectos para los Estados-nación de América del Norte relajar sus controles fronterizos para que decrezca la inmigración irregular o - como mínimo - para que se establezcan prioridades comunes en la política migratoria, parece improbable que dicho desarrollo ocurra en un futuro próximo. No obstante, la idea de una «Unión Americana» permanece como un ideal y una opción que puede, en el futuro, ser una solución más viable para establecer alguna forma de control sobre la inmigración irregular en América del Norte.

\section{LA INCLUSIÓN DE LAS NACIONES DE AMÉRICA CENTRAL Y SUDAMÉRICA EN UNA UNIÓN AMERICANA}

Si una Unión Americana llegase a hacerse realidad, la siguiente cuestión sería si - y cómo- cualquier Estado-nación de América Central o de Sudamérica podría o debería unirse a la alianza. La inclusión de países de América Central y Sudamérica en una Unión Americana tendría diversas implicaciones para todo el continente, tanto positivas como negativas. La consecuencia más obvia de una Unión Americana que se extienda desde el norte de Canadá a Tierra del Fuego sería la potencialidad de grandes migraciones de personas desde las naciones miembros más pobres y más inestables, tales como El Salvador, Honduras y Guatemala, a las naciones miembros más ricas y más estables, tanto en América del Norte como en Sudamérica.

Dicha migración a gran escala entre naciones miembros desigualmente situadas ocurrió en la Unión Europea, como resultado de la doctrina de la libertad de circulación, y ciudadanos de naciones miembros de Europa central y oriental emigraron a prósperos Estados miembros, tales como Irlanda y otros Estados-nación de Europa occidental y

\footnotetext{
57 Véase, p. ej., PRAMUK, J., «Trump: I'm building the wall even if we have to close down our government», CNBC, 22 agosto 2017, disponible en https://www.cnbc.com/2017/08/22/trump-says-heswilling-to-shut-down-the-government-to-get-his-border-wall.html.

${ }^{58}$ En la UE, la migración irregular dentro de Europa decreció entre 2007 y 2010. Véase MOREHOUSE, C. y BLOMFIELD, M., Irregular Migration in Europe, Migration Policy Institute (Washington, D.C., 2011), disponible en https://www.migrationpolicy.org/pubs/TCMirregularmigration.pdf, en 3.
} 
septentrional $^{59}$. Como resultado, sigue existiendo un gran acuerdo de tensión entre ciudadanos de diversos Estados miembros, por causa del constante flujo de ciudadanos entre Estados miembros de la UE que buscan un mejor trabajo y otras oportunidades de calidad de vida ${ }^{60}$.

Dicho desequilibrio entre los Estados-nación más pobres de América Central y los países más prósperos de América del Norte y Sudamérica, produciría probablemente que América Central y/o Sudamérica fuesen invitadas a formar parte de una «Unión Americana». No es difícil imaginar incluso grandes cifras de migrantes de América Central, en particular, desplazándose a sus vecinos tanto en América del Norte como a Sudamérica, con la finalidad de buscar nuevas oportunidades laborales y, en consecuencia, una vida mejor para ellos mismos y sus familias. Esto probablemente daría lugar a un tipo de tensión y resentimiento crecientes en las Américas, que ya ha sido visto en Europa entre los diversos Estados miembros. Sin embargo, si una «Unión Americana» llegase a convertirse en una posibilidad seria, la idea de expandir las oportunidades de afiliación a al menos algunos de los Estados-nación de América Central y Sudamérica debería considerarse seriamente, si uno de los objetivos para establecer dicha colectividad fuese regular el flujo de inmigrantes irregulares en las Américas.

\section{CONCLUSIÓN: EL FUTURO DEL ESTADO-NACIÓN}

En los albores del siglo XXI, ¿la era del Estado-nación parece que se acaba? Cuando se firmó el Tratado de Maastricht el 7 febrero 1992, menos de 50 años después del fin de la II Guerra Mundial ${ }^{61}$, parecía como si Europa y, en verdad, el mundo estuviesen en la senda de la paz, la prosperidad y la unidad, a través de la recíproca colaboración voluntaria entre Estados-nación ${ }^{62}$. A pesar de las controversias y preocupaciones políticas recientes que rodean a los migrantes irregulares que buscan refugio en Europa, la Unión Europea permanece como una alianza poderosa de Gobiernos fuertes de Estados-nación, que intentan trabajar juntos para alcanzar unidos su compromiso colaborativo recíproco ${ }^{63}$.

Queda por ver si el resto del mundo seguirá o no el ejemplo de la Unión Europea, y buscará establecer controles fronterizos más cooperativos y relajados. Entre tanto, nos corresponde a nosotros en el resto del mundo $-\mathrm{y}$ particularmente, a nosotros en América del Norte- decidir si nuestro futuro incluye un compromiso de establecer una

59 Véase, p. ej., Migration Watch UK, disponible en https://www.migrationwatchuk.org/keytopics/european-union (última actualización, septiembre 2015) («El número de europeos del este en el Reino Unido ha aumentado a cerca de un millón desde 2004»).

${ }^{60}$ Ibidem.

${ }^{61}$ Véase EUROPEAN CENTRAL FUND, «Five things you need to know about the Maastricht Treaty», 15 febrero 2017, disponible en https://www.ecb.europa.eu/explainers/tell-memore/html/25_years_maastricht.en.html.

${ }^{62}$ Véase GRIVEAUD, M., «Why is the Maastricht Treaty considered to be so significant?», 29 mayo 2011, disponible en http://www.e-ir.info/2011/05/29/why-is-the-maastricht-treaty-considered-to-beso-significant-2/.

${ }^{63}$ Véase SURI, J., «Why the EU Still Matters in a Fracturing World», Fortune, 16 diciembre 2016, disponible en http://fortune.com/2016/12/14/european-union-populism-globalization-refugees/. 
ciudadanía global, o si los temores al terrorismo darán lugar a un aislamiento creciente y a una militarización de las fronteras internacionales.

\section{BIBLIOGRAFÍA}

- AMERICAN IMMIGRATION COUNCIL, «How DACA is Impacting the Lives of Those Who are Now DACAmented», Informe especial, 15 agosto 2013, disponible en https://www.americanimmigrationcouncil.org/research/how-daca-impacting-lives-thosewho-are-now-dacamented.

- CAMPBELL, K.M., "Imagining a more humane immigration policy in the age of Obama: The use of plenary power to halt the State balkanization of immigration regulation», Saint Louis University Public Law Review, núm. 29 (2010).

- CLARK, D., «House Passes “Kate's Law”, Votes to Defund Sanctuary Cities», NBC News, $29 \quad$ junio 2017, disponible en https://www.nbcnews.com/politics/immigration/house-immigration-crackdown$\underline{\mathrm{n} 778141 .}$.

- CNN WIRE STAFF, «GOP Roars Back to Take U.S. House; Democrats Cling to Senate Majority», CNN, 3 noviembre 2010, disponible en http://www.cnn.com/2010/POLITICS/11/02/election.main/index.html.

- COLLINSON, S., «Republicans seize Senate, gaining full control of Congress», CNN, 5 noviembre 2014, disponible en http://www.cnn.com/2014/11/04/politics/election-day-story/index.html.

- DEARDEN, L., «Refugee Crisis: Concern over "unprecedented" arrivals in Greece and Italy after 2016 total passes 100,000», Independent, 23 febrero 2016, disponible en http://www.independent.co.uk/news/world/europe/refugee-migrant-crisis-concernunprecedented-numbers-greece-italy-2016-passes-100000-a6891101.html.

- ESTERBROOK, J., «Voters Usher Out Republicans», CBS News, 18 diciembre 2016, disponible en https://www.cbsnews.com/news/voters-usher-out-republicans/.

- EUROPEAN CENTRAL FUND, «Five things you need to know about the Maastricht Treaty», 15 febrero 2017, disponible en https://www.ecb.europa.eu/explainers/tell-me-more/html/25_years_maastricht.en.html.

- EUROPEAN COMMISSION, «Freedom to live and move in Europe: A guide to your rights as an EU citizen», EU Law and Publications, 13 octubre 2017, disponible en https://publications.europa.eu/en/publication-detail/-/publication/51cf67c4-a4ed-44a693cf-8120a7aa4c08/language-en/format-PDF/source-search.

- EUROPEAN COMMISSION, «Freedom to move and live in Europe: A Guide to your rights as an EU citizen», EU Law and Publications, 17 octubre 2013, disponible en 
https://publications.europa.eu/en/publication-detail/-/publication/51cf67c4-a4ed-44a693cf-8120a7aa4c08/language-en/format-PDF/source-search.

- FOLEY, E., «Over Half the States are Suing Obama For Immigration Actions», The Huffington Post, 25 enero 2015, disponible en https://www.huffingtonpost.com/2015/01/26/states-lawsuitimmigration_n_6550840.html.

— FORD, M., «A Ruling Against the Obama Administration on Immigration», The Atlantic, 10 noviembre 2015, disponible en https://www.theatlantic.com/politics/archive/2015/11/fifth-circuit-obamaimmigration/415077/.

— GILBERT, J., Nomadic Peoples and Human Rights, Routledge (Londres, 2014).

- GONZALES, R., «Trump Travel Ban Allowed To Take Partial Effect», The Two Way: Breaking News from NPR, 13 noviembre 2017, disponible en https://www.npr.org/sections/thetwo-way/2017/11/13/563923136/trump-travel-banallowed-to-take-partial-effect.

— GORDON, I. y RAJA, T., «164 Immigration Laws Passed Since 2010? A MoJo Analysis», Mother Jones, marzo/abril 2012, disponible en http://www.motherjones.com/politics/2012/03/anti-immigration-law-database/\#.

- GRIVEAUD, M., «Why is the Maastricht Treaty considered to be so significant?», 29 mayo 2011, disponible en http://www.e-ir.info/2011/05/29/why-is-the-maastrichttreaty-considered-to-be-so-significant- $2 /$.

- GROENENDIJK, K., GUILD, E. y CARRERA, S. (Editores), Illiberal Liberal States: Immigration, Citizenship and Integration in the EU, Routledge (Londres, 2013).

- HATTEM, J., «France declares state of emergency, closes borders after attacks», The Hill, 13 noviembre 2015, disponible en http://thehill.com/policy/nationalsecurity/260134-france-declares-state-of-emergency-closes-borders-after-attacks.

— INTERNATIONAL RESCUE COMMITTEE, «Refugee Crisis: Europe and Middle East», disponible en https://www.rescue.org/topic/refugee-crisis-europe-middle-east.

- KRISHNAKUMAR, P., FOX, J. y LEVINE, A., "What's next for DACA and the nearly 800,000 people protected by it», Los Angeles Times, 6 septiembre 2017, disponible en http://www.latimes.com/projects/la-na-pol-daca-future/.

- KUPPERMAN, K.O., North America and the beginnings of European colonization, American Historical Association (Washington, D.C., 1992).

- MASON, J., «Trump seeks border wall, crackdown on unaccompanied minors for "Dreamer" deal», Reuters, 8 octubre 2017, disponible en https://www.reuters.com/article/us-usa-immigration-exclusive/trump-Véaseks-borderwall-crackdown-on-unaccompanied-minors-for-dreamer-deal-idUSKBN1CD10L. 
- MCKINLEY Jr., J.C., «A Mexican Manual for Illegal Migrants Upsets Some in U.S.», The New York Times, 6 enero 2005, disponible en http://www.nytimes.com/2005/01/06/world/americas/a-mexican-manual-for-illegalmigrants-upsets-some-in-us.html.

- MOHDIN, A., «These are the routes being closed off to refugees fleeing into Europe», Quartz, 10 marzo 2016, disponible en https://qz.com/635110/these-are-theroutes-being-closed-off-to-refugees-fleeing-into-europe/.

- MOREHOUSE, C. y BLOMFIELD, M., Irregular Migration in Europe, Migration Policy Institute (Washington, D.C., 2011), disponible en https://www.migrationpolicy.org/pubs/TCMirregularmigration.pdf.

- MURRAY, S. y MONTGOMERY, L., «House passes health-care reform bill without Republican votes», The Washington Post, 22 marzo 2010, disponible en http://www.washingtonpost.com/wpdyn/content/article/2010/03/21/AR2010032100943.html.

- O'SUlLIVAN, F., «The Fading Dream of a Borderless Europe», Citylab, 13 enero 2017, disponible en https://www.citylab.com/equity/2017/01/the-fading-dream-of-aborderless-europe/512816/.

- PRAMUK, J., «Trump: I'm building the wall even if we have to close down our government», $C N B C, 22$ agosto 2017, disponible en https://www.cnbc.com/2017/08/22/trump-says-hes-willing-to-shut-down-thegovernment-to-get-his-border-wall.html.

- PRESTON, J. y CUSHMAN, Jr., J.H., «Obama to Permit Young Migrants to Remain in U.S.», The New York Times, 15 junio 2012, disponible en http://www.nytimes.com/2012/06/16/us/us-to-stop-deporting-some-illegal-

immigrants.html.

- RODRIGUEZ, C.Y., «Latino vote key to Obama's re-election», CNN, 9 noviembre 2012, disponible en http://www.cnn.com/2012/11/09/politics/latino-vote-keyelection/index.html.

- ROGERS, J., «Europe flooded with over 1,700 ISIS terrorists primed to attack, EU reveals», Express, 9 diciembre 2016, disponible en https://www.express.co.uk/news/world/741587/ISIS-terrorists-EU-Europe-flooded.

— SATI, J., «How DACA pits "good immigrants" against millions of others», The Washington Post, 7 septiembre 2017, disponible en https://www.washingtonpost.com/news/posteverything/wp/2017/09/07/how-daca-pitsgood-immigrants-against-millions-of-others/?utm_term $=.85 \mathrm{~b} 6 \mathrm{f} 446689 \mathrm{~b}$.

- SHEAR, M.D. y DAVIS, J.H., «Trump Moves to End DACA and Calls on Congress to Act», The New York Times, 5 septiembre 2017, disponible en 
https://www.nytimes.com/2017/09/05/us/politics/trump-daca-dreamersimmigration.html.

- SHERRY, A., «Illegal-immigrant children brought to U.S. get break from deportation», The Denver Post, 15 junio 2012, disponible en http://www.denverpost.com/2012/06/15/illegal-immigrant-children-brought-to-u-s-getbreak-from-deportation/.

— SURI, J., «Why the EU Still Matters in a Fracturing World», Fortune, 16 diciembre 2016, disponible en http://fortune.com/2016/12/14/european-union-populismglobalization-refugees/.

— TREVINO, R., «DACA and "dreamers" worthy of support», USA Today, 30 octubre 2017, disponible en https://www.usatoday.com/story/opinion/contributors/2017/10/30/daca-and-dreamersworthy-support/807131001/.

- UNITED NATIONS HIGH COMMISSIONER ON REFUGEES, «Note on NonRefoulement (Submitted by the High Commissioner)», disponible en http://www.unhcr.org/en-us/excom/scip/3ae68ccd10/note-non-refoulement-submittedhigh-commissioner.html.

- WEINER, R., «How immigration reform failed, over and over», The Washington Post, 30 enero 2013, disponible en https://www.washingtonpost.com/news/thefix/wp/2013/01/30/how-immigration-reform-failed-over-andover/?utm_term=.e16bbbb89dd4.

- WESTCOTT, L., «The World's 'Unprecedented' Refugee Crisis By the Numbers», Newsweek, 18 junio 2015, disponible en http://www.newsweek.com/worldsunprecedented-refugee-crisis-numbers-344171.

- WHEELER, L. y FABIAN, J., «Deadlocked Supreme Court blocks Obama on immigration», The Hill, 23 junio 2016, disponible en http://thehill.com/regulation/courtbattles/president-obama-immigration-actions-programs-blocked-supreme-courtdeadlocked-scalia-dapa-daca-crushing-blow.

- WONG, S. y TOEPLITZ, S., «DREAM Act Dies in Senate», Politico, 20 diciembre 2010, disponible en https://www.politico.com/story/2010/12/dream-act-dies-in-senate$\underline{046573}$.

- YELLIN, J., «Obama not pushing to pass immigration reform this year», $C N N, 10$ abril 2009, disponible en http://www.cnn.com/2009/POLITICS/04/09/obama.immigration/. 